\title{
Erratum to: Predictive Diagnosis of Glaucoma Based on Analysis of Focal Notching along the Neuro-Retinal Rim Using Machine Learning
}

\author{
Rishav Mukherjee ${ }^{a, *}$, Shamik Kundu ${ }^{a}$, Kaushik Dutta ${ }^{a}$, Anindya Sen $^{a}$, and Somnath Majumdar ${ }^{b}$ \\ ${ }^{a}$ Department of Electronics and Communication Engineering, Heritage Institute of Technology, Kolkata, India \\ ${ }^{b}$ Department of Ophthalmology, Apollo Gleneagles Hospital, Kolkata, India \\ *e-mail: rishavmukherjee17@gmail.com
}

DOI: $10.1134 / \mathrm{S} 1054661819040230$

The original article can be found online at https://doi.org/10.1134/S1054661819030155

Page 532, right column, the photos and information about authors Anindya Sen and Somnath Majumdar should read:

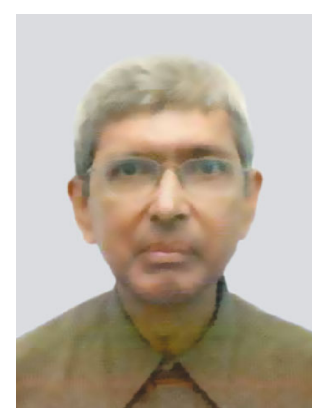

Anindya Sen is a Professor at the department of electronics and communication engineering, Heritage Institute of Technology, a private autonomous engineering college in Anandapur, Kolkata, India. He received his B.E. from Jadavpur University, India in the year 1980, PhD from University of Minnesota, Twin Cities in 1996, and got his Post-Doctoral training from University of Chicago from 1996 to 2000. He currently holds one US

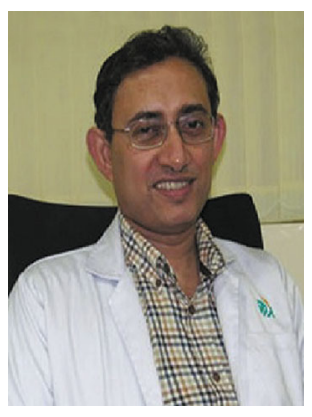
Somnath Majumdar is a senior consultant ophthalmologist in Kolkata, India for last 20 years. He is currently a consultant ophthalmologist for Apollo Hospitals, Kolkata and Fortis Hospitals. He had done post graduation from Dr. R.P. Centre for Ophthalmic Sciences, AIIMS, New Delhi and completed his FRCS (Edin. and Glasgow) in 2000. He is an expert in fields of glaucoma, cataract, and retinal surgery.

patent and thirty research paper publications. His research interests include, medical image processing, Internet of things, artificial intelligence, and VLSI design.

Received November 14, 2018; revised April 11, 2019; accepted April 17, 2019 